\title{
Comparative multivariable statistical analysis of the dynamics of motor vehicles when they move in urban and extraurban environments
}

\author{
Cătălin-Dumitru Darie ${ }^{1}$, Ion Copae ${ }^{2}$ \\ Eng., S.A-R City Insurance, Bucharest, Romania ${ }^{1}$ \\ Professor eng. PhD, Department of Military Vehicles and Transportation, Military Technical Academy "Ferdinand I", \\ Bucharest, Romania ${ }^{2}$
}

\begin{abstract}
Based on multivariable statistical analysis, the paper presents some functional particularities of the dynamics of vehicles when moving them in urban and extraurban areas. There are several aspects related to the study of vehicle dynamics by applying the methods of multivariable statistics. The study is based on experimental data obtained from tests of Ford Focus and Volkswagen Touareg II 3.0 V6 TDI cars equipped with Diesel engine, as well as tests of Logan Laureate car equipped with gasoline injection engine, all equipped with on-board computer, sensors and built-in actuators.

The concepts and algorithms specific to multivariable statistics are presented, which are applied in the case of large data sets, as is the case of vehicles equipped with a computer on board. For this purpose, spatial correlation analysis, canonical correlation analysis, principal component analysis, factor analysis, group analysis, discriminatory analysis, data paternity classification and multidimensional scaling are used. These processes have ensured the detection of central trends in evolution, reduced data and better readability by reducing the space with more dimensions in the plan. Comparative conclusions are drawn on the dynamics of motor vehicles in urban and extraurban areas.
\end{abstract}

Keywords: vehicle dynamics, multivariable statistics, spatial correlation, canonical correlation analysis, principal component analysis.

\section{INTRODUCTION}

This paper aims to establish functional features based on multivariable statistical algorithms [3], when moving vehicles in urban and extraurban areas, where traffic conditions are usually different. Addressing the dynamics of vehicles has been one of the main concerns of specialists in the field, who have constantly sought to improve the dynamic and economical performance of road transport. The developments of different disciplines in various fields, the emergence of more and more advanced experimental equipment and the equipment of vehicles with electronic control systems were the most important factors influencing the study algorithms of their dynamics. Through specific methods and algorithms, multivariable statistics allow highlighting important functional aspects theoretically and practically.

\section{EXPERIMENTAL RESEARCH}

To perform comparative multivariable analysis, experimental research was conducted with a Ford Focus car and a Volkswagen Touareg II 3.0 V6 TDI car, both equipped with supercharged diesel engine and common-rail fuel supply system. Experimental research was also carried out with a Dacia Logan Laureate car equipped with a petrol injection engine.

For the acquisition of data from the on-board computer, the Ford interface and software were used. For Volkswagen Touareg was used the VCDS application developed by Ross-Tech, which is the specialized diagnostic software for the Volkswagen group. For the Logan Laureate car, the Clip tester and the related software from Renault were used. From the purchased data, 40 samples from the urban environment and 40 samples from the extraurban environment were retained for each car, more significant for the purposes pursued $[9,10,11]$.

For example, the upper graphs in fig. 1 show the position of the accelerator pedal $\mathrm{p}$ (considered the engine load), and the lower graphs the engine speed $n$.

As can be seen from fig. 1, the values of the load and engine speed are higher in the extraurban environment than in the urban environment. Thus, from the upper graphs it is observed that the engine load varied in the range of $5-86.6 \%$ in urban areas and in the range of 6.6-98.4\% in extraurban areas; the average for all samples was $10.2 \%$ higher in the extraurban environment compared to the urban one. 


\section{International Advanced Research Journal in Science, Engineering and Technology}

Vol. 8, Issue 4, April 2021

\section{DOI: 10.17148/IARJSET.2021.8417}

From the lower graphs in fig. 1 it is observed that the engine speed varied in the interval 917.4-2910.1 rpm in the urban environment and in the interval 1172.6-3885.6 rpm in the extraurban environment; the average of all the samples was higher by $1007.6 \mathrm{rpm}$ in the extraurban environment compared to the urban one.

\section{Accelerator pedal position and engine speed,}

40 tests in urban areas and 40 tests in extraurban areas, Volkswagen Touareg II 3.0 V6 TDI car

a) Accelerator pedal position, urban environment

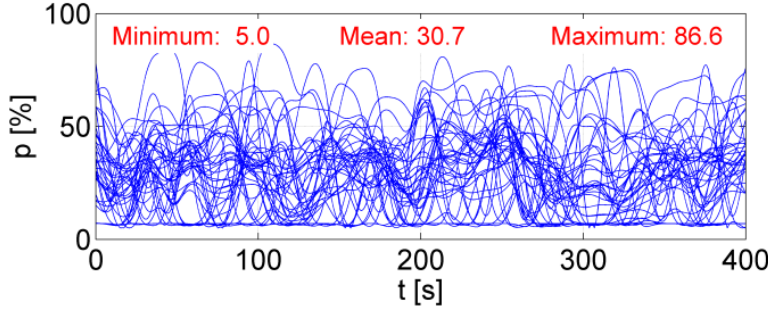

c) Engine speed in the urban environment

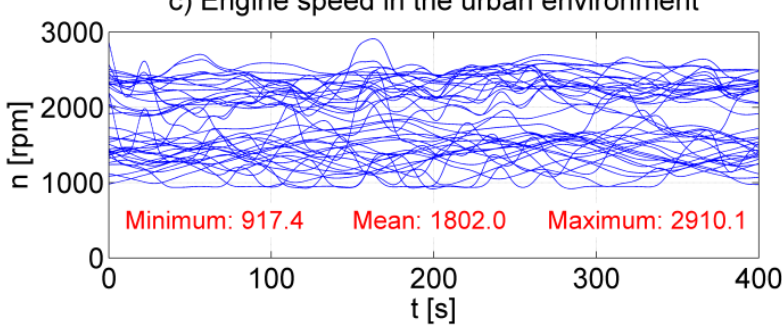

b) Accelerator pedal position, extraurban environment

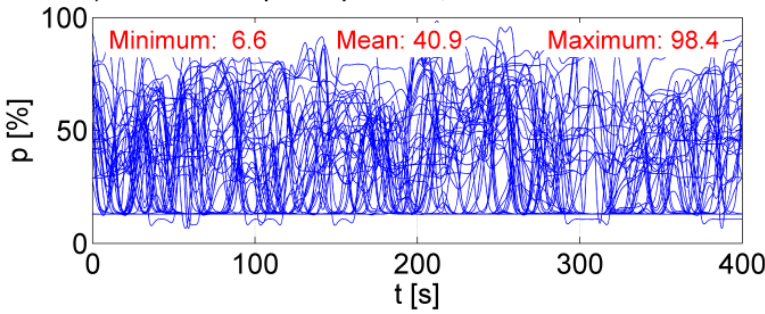

d) Engine speed in the extraurban environment

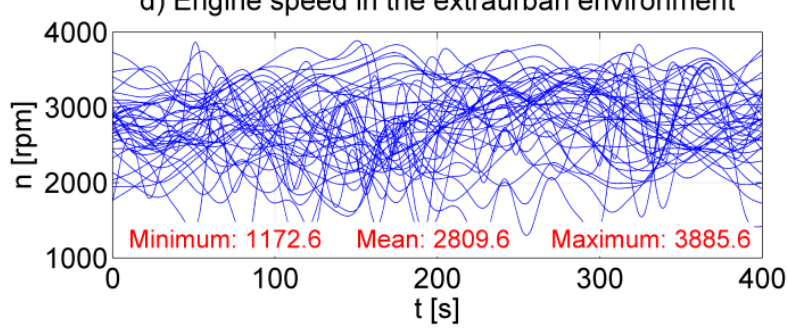

Fig. 1 Accelerator pedal position and engine speed, Volkswagen Touareg II 3.0 V6 TDI car

The graphs on the left in fig. 2 show the average values on speed $V$ tests, and the graphs on the right show the maximum values on speed tests. The upper graphs show the values in the urban environment, and the lower graphs in the extraurban environment.

As can be seen in fig. 2, the average and maximum values on speed tests are higher in the extraurban environment than in the urban environment. For example, the graphs on the left show that the speed varied between 30.2-48.6 km/h in urban areas and between $90.4-121.4 \mathrm{~km} \mathrm{~h}$ in extraurban areas; the average of all the tests was 2.51 times higher in the extraurban environment compared to the urban one.

Average and maximum values on speed tests, Volkswagen Touareg II 3.0 V6 TDI car

a) Urban 40 tests, average values

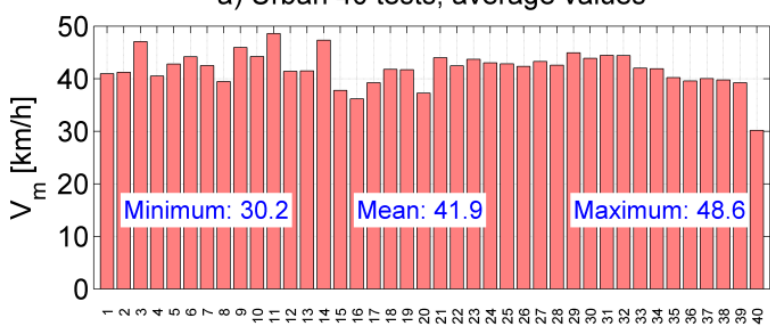

c) Extrarban 40 tests, average values

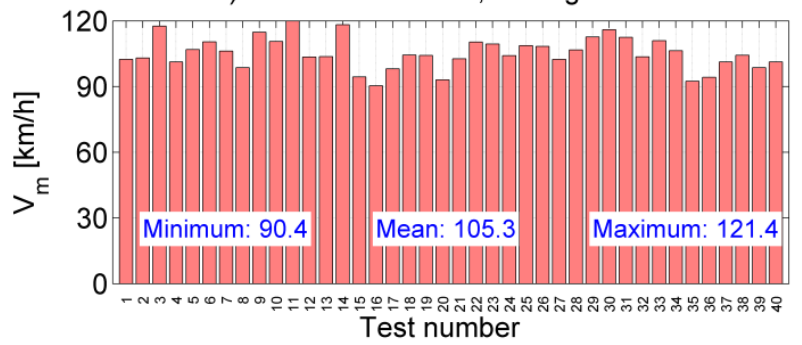

b) Urban 40 tests, maximum values

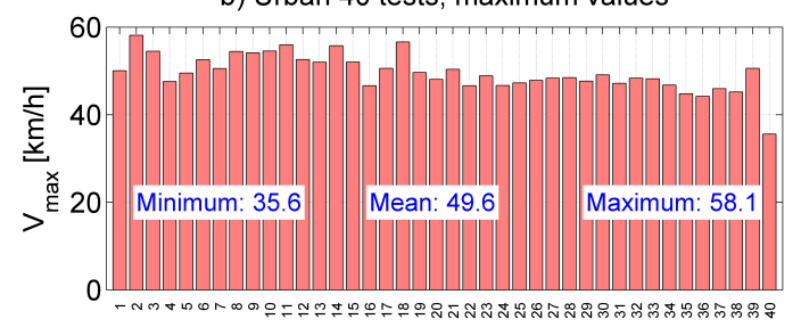

d) Extraurban 40 tests, maximum values

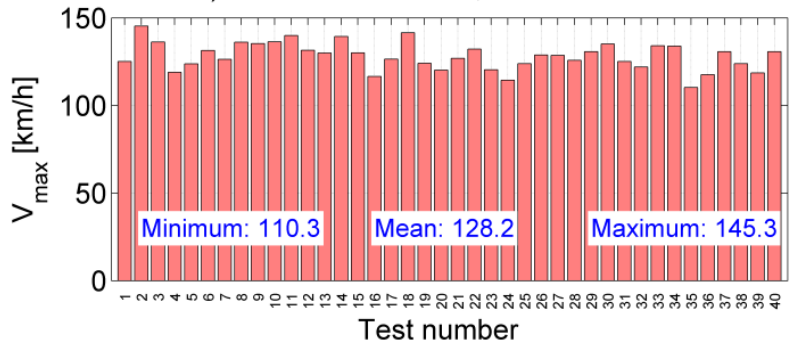

Fig. 2 Average and maximum values on speed tests, Volkswagen Touareg II 3.0 V6 TDI car 


\section{International Advanced Research Journal in Science, Engineering and Technology}

Vol. 8, Issue 4, April 2021

\section{DOI: 10.17148/IARJSET.2021.8417}

\section{III.MULTIVARIATE STATISTICAL ANALYSIS}

Multivariable statistics, also known as spatial statistics or geostatistics, represents a component of statistics which targets large sets of data, with time variation, like in case of classical statistics, and with space variation, from which it derives the last two names; first name is due to the analysis of different types of data, meaning multiple variables [3, 7]. Multivariable statistics can be applied in the automotive field due to the advent of engine's electronic control of functioning, where, by the existence of a group of senzorial parameters, the on-board computer operates with large sets of data, received from sensors and actuators embedded from factory.

Multivariable statistics uses analysis, concepts and specific algorithms. Therefore, plane correlation specific to classical statistics becomes spatial correlation, adding itself a third dimension, meaning the distance, which defines geostatistics. A special form represents the canonical correlation analysis, which is related to multivariable mathematical models. Likewise, multivariable statistics uses group analysis, defining which is common to data, as well as discriminant analysis, which targets the differences between data.

Because humans cannot detect variations within the space with more than three dimensions, geostatistics uses transformations which ensure the replacement of initial multivariable picture with equivalent ones, so that the graphical plots becomes tri-spatial, the most; this is specific to the analysis based on main components. In order to conclude after data analysis, multivariable statistics also uses factor based analysis, as well as classification procedures, related to decision theory.

Follow-up, there are presented some elements specific to multivariate statistics, in order to highlight other particularities of vehicle dynamics, for urban and extraurban environment.

\section{A. Spatial correlation analysis}

As it is known, time correlation represents a classic correlation; on the other hand, spatial correlation also uses the distance, which is a parameter characteristic to multivariate statistics. As a consequence, spatial correlation shows how much the influence on a certain point on space depends on the values of surrounding ones. In general, a positive spatial correlation shows that similar values tend to close in; a negative spatial correlation depicts a tendency of values to spread $[6,8]$. coefficient.

As it is known, classical time correlation is assessed based on correlation coefficient $\rho$, also called Pearson

For the quantitative assessment of the spatial correlation, two indices are used, Moran's coefficient and Geary's coefficient, where the latter is calculated with the expression:

$$
G=\frac{n-1}{2 S} \frac{\sum_{i=1}^{n} \sum_{j=1}^{n} w_{i j}\left(x_{i}-x_{j}\right)^{2}}{\sum_{i=1}^{n}\left(x_{i}-\bar{x}\right)^{2}}
$$

which is also denoted by c, Geary's coefficient marks a local spatial correlation, and Moran's coefficient a global spatial correlation.

In this formula there are noted: $x i$ and $x j$ - random variable values $x$ (with $n$ values) being in the same class at distances $\mathrm{i}$ and $\mathrm{j}$ of center; $\bar{x}$ - average value of variable $\mathrm{x}$; wij - weight factor matrix, in which value 1 shows that the pair $(x i, x j)$ is in the same class, and the null value indicates the opposite (that is why it is also called proximity or contingency table); $\mathrm{S}$ - sum of elements wij of the same class.

Geary's coefficient varies in the range $G \in[0 ; 2]$; the null value indicates a perfect positive correlation, the value 2 a perfect negative correlation, and the value +1 the absence of any correlation (total independence).

As an example, in fig. 3 shows the values of Geary's coefficient for the speed of vehicle V (upper graphs) and for the engine moment Me (lower graphs) for the Ford Focus car. Similarly, in fig. 4 shows the values of Geary's coefficient for the acceleration of the vehicle a (upper graphs) and for the fuel consumption per $100 \mathrm{~km}$ traveled C100 (lower graphs) for the Logan Laureate car.

The graphs in fig. 3 and fig. 4 shows that all values of the Geary's coefficient are different from the unit value, so there is always a spatial correlation. In addition, with subunit and supraunit values, it follows that there are positive correlations and negative correlations.

From fig. 3 and fig. 4 it also results that in the extraurban environment the values are higher than in the urban environment; therefore, when moving in the extraurban environment, the spatial correlation is more accentuated than in the urban one. 
DOI: $10.17148 /$ IARJSET.2021.8417

Local spatial correlation, Geary`s coefficient, $\mathbf{4 0}$ tests in urban and $\mathbf{4 0}$ in extraurban environment, Ford Focus car

a) Vehicle speed, urban environment

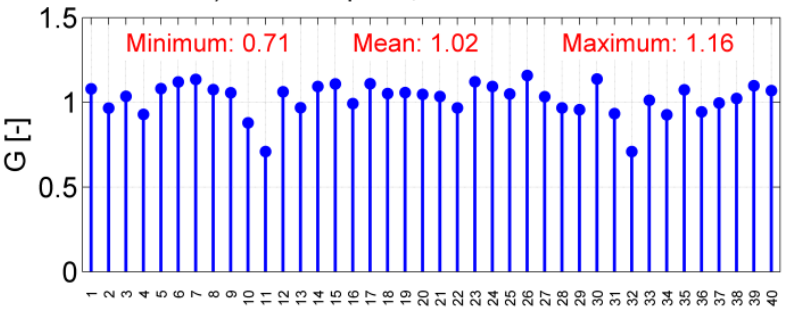

c) Engine power, urban environment

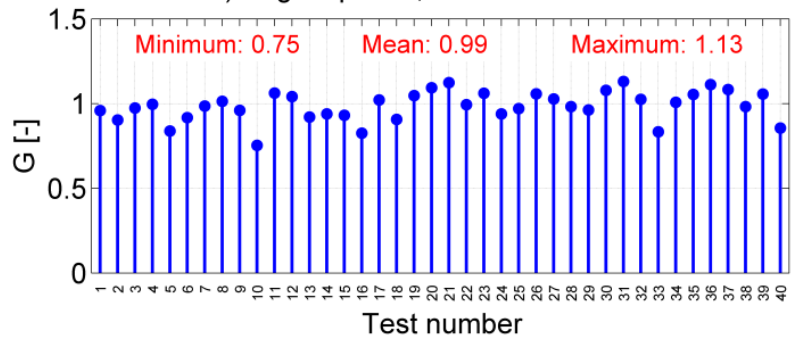

b) Vehicle speed, extraurban environment

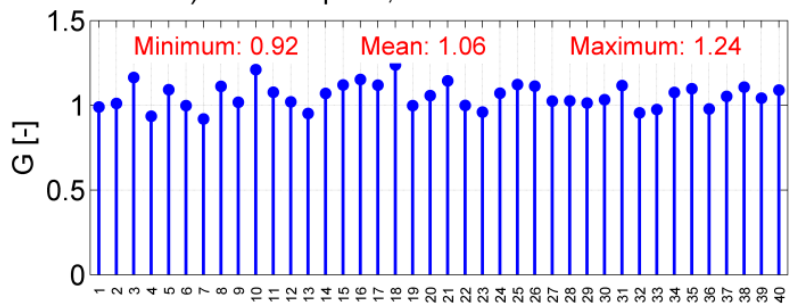

d) Engine power, extraurban environment

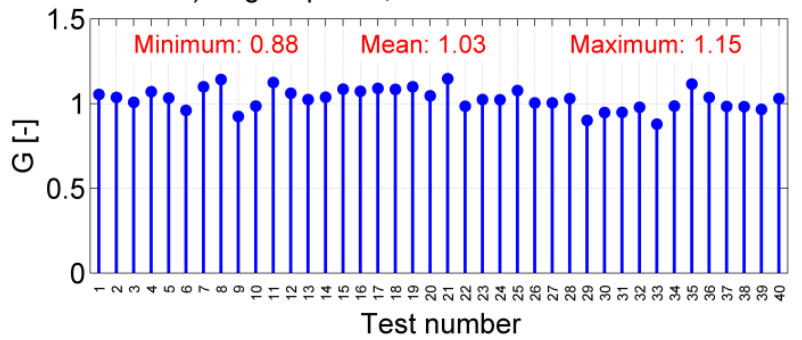

Fig. 3 Local spatial correlation, Geary's coefficient, Ford Focus car

Local spatial correlation, Geary`s coefficient, $\mathbf{4 0}$ tests in urban and $\mathbf{4 0}$ in extraurban environment, Logan Laureate car

a) Vehicle acceleration, urban environment

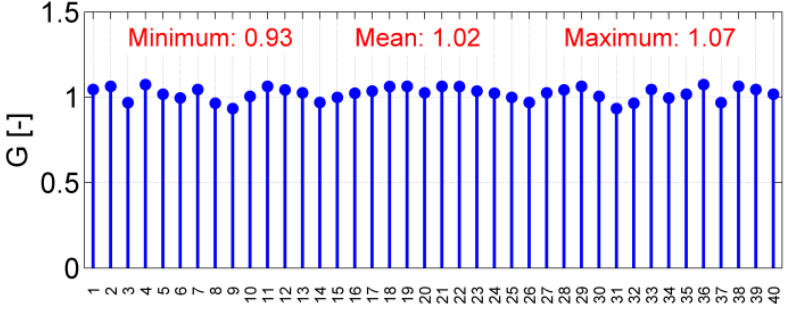

c) Fuel consumption per $100 \mathrm{~km}$, extraurban environment

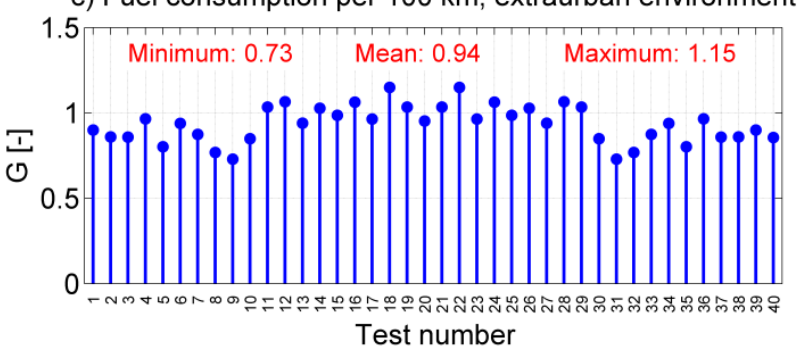

b) Vehicle acceleration, extraurban environment

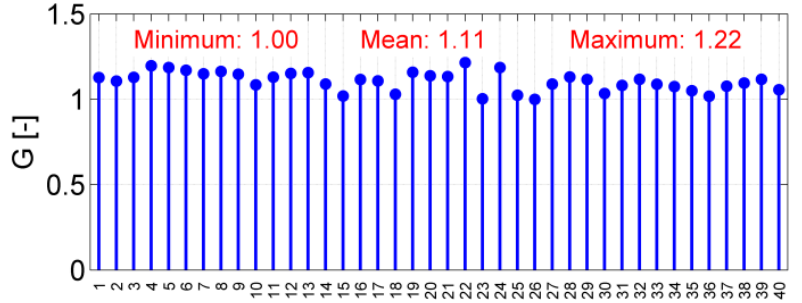

d) Fuel consumption per $100 \mathrm{~km}$, extraurban environment

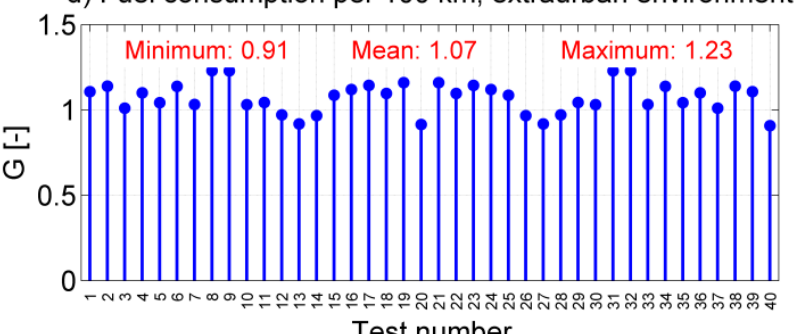

Fig. 4 Local spatial correlation, Geary's coefficient, Logan Laureate car

B. Principal component analysis

The analysis on principal components is part of the category of multivariable techniques that allow the reduction of the data size, ensuring the highlighting of the central tendencies of evolution of a process. Mathematically expressed, principal component analysis (PCA) allows the transformation of a set of correlated quantities in space that has $m$ dimensions, into a set of uncorrelated variables in space that has $d$ dimensions $(d<m)$.

Unrelated quantities, called principal components, are linear combinations of the original correlated variables [4]. In the case of motor vehicles, where the on-board computer operates with large data sets, it becomes necessary to reduce the size of the data. Transforming a data set is equivalent to rotating them; in the case of PCA, the rotation of the original data is ensured so as to ensure a maximum variance of them on the principal axes (PA) which are orthogonal. In this way, a set of correlated variables is transformed into a set of uncorrelated quantities that are ordered in the sense of decreasing the variability (dispersion). Consequently, the first principal component PC1, designed on the pricipal axis PA1, corresponds to the largest variation of the data (fig. 5 with 800 values); the second principal component PC2, 


\section{International Advanced Research Journal in Science, Engineering and Technology}

Vol. 8, Issue 4, April 2021

\section{DOI: $10.17148 /$ IARJSET.2021.8417}

designed on the principal axis PA2, is related to the next variation of the data in descending order etc. Arranging on the principal axes which are orthogonal, so the scalar product of the principal components being zero, they are uncorrelated.

As can be seen from fig. 5, in the direction of the first principal axis PA1 the variation of the data is the largest; in the direction of the second principal axis PA2 the variation of the data is smaller than in the first case. In both graphs is shown the ellipse of $98 \%$ confidence.

Principal components analysis and establishment of principal axes

a) Initial data and principal axes

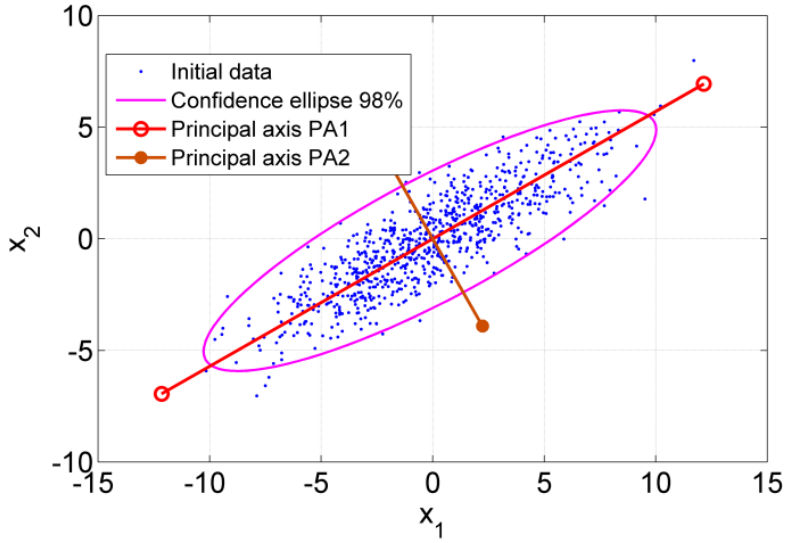

b) Rotated data and principal axes

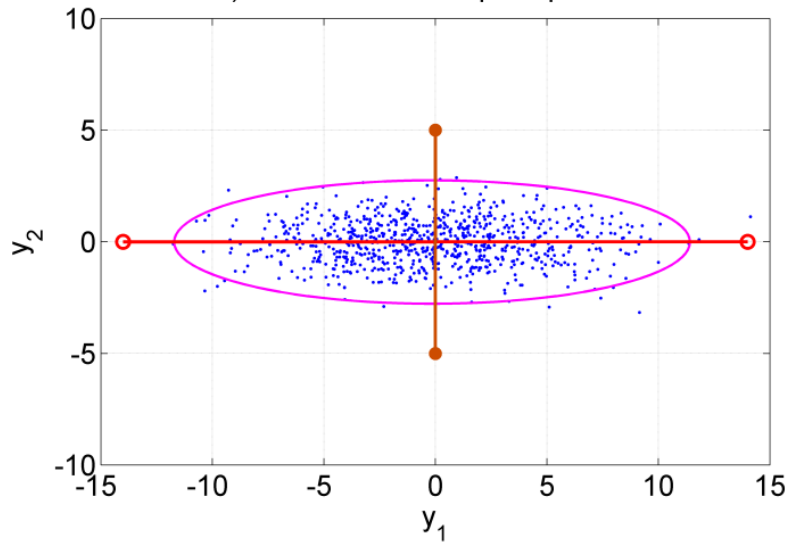

Fig. 5 Principal component analysis and establishment of principal axes for a bivariate parameter

There are several procedures used by PCA; the most used uses matrix methods. In this case, the original (initial) data are used to calculate the covariance matrix, which has the dispersions on the main diagonal. For this purpose, we proceed to establish the eigenvectors and eigenvalues of the covariance matrix; the eigenvectors correspond to the directions of the principal axes and the eigenvalues constitute the individual contributions of the principal components to the total dispersion. As it can be seen, the PCA uses the variations of the analyzed quantities, namely the dispersions, which give the measure of the deviations of the variables from their average values.

It is further considered that there is a set $\mathrm{X}$ of $n$ observations $\mathbf{x}$, each in the $m$-dimensional space, in which the vectors $\mathbf{x}$ are centered, so with zero mean (from the initial variables the average is extracted to notice the dispersion, so the variation). The principal component analysis establishes a set of orthogonal vectors $\mathbf{v}_{i}$, called principal vectors, ordered in descending order of the value of the dispersion. The initial data are projected on the principal axes (given by orthogonal vectors $\mathbf{v}_{i}$ ), so the PCA ensures the transformation:

$$
\mathbf{y}_{i}=\mathbf{v}_{i}^{t} \mathbf{x}_{i}
$$

with the symbol $t$ marking the mathematical operation of transposition.

The first principal vector $\mathbf{v}_{1}$ corresponds to the largest dispersion, so the principal axis PA1. The second main vector $\mathbf{v}_{2}$ ensures the maximization of the dispersion in the direction orthogonal to the vector $\mathbf{v}_{1}$, corresponding to the principal axis PA2 etc. As a result, the final solution of the PCA ensures the establishment of the eigenvectors and eigenvalues of the covariance matrix $\mathbf{C}_{x}$ given by the expression:

$$
\mathbf{C}_{x}=\frac{1}{n-1} \mathbf{x}^{t} \mathbf{X}=\mathbf{V} \mathbf{\Lambda} \mathbf{v}^{t}
$$

where the eigenvectors are:

$$
\mathbf{V}=\left[\begin{array}{llll}
\mathbf{v}_{1} & \mathbf{v}_{2} & \ldots & \mathbf{v}_{m}
\end{array}\right]
$$

and the matrix $\Lambda(m \times m)$ contains the real and non-negative eigenvalues arranged in descending order $\left(\lambda_{1}>\lambda_{2}>\ldots>\lambda_{m}\right)$. Therefore, the descending order of the dispersions corresponds to the descending order of the eigenvalues.

According to the presented, it results that the number of principal components is equal to the number of initial variables. However, the purpose of the PCA is to reduce the number of principal components, so that the subsequent analysis is limited only to those that highlight the largest variations in size in the directions of the principal axes. Therefore, for the initial variables only the principal components corresponding to the largest variations (dispersions) are retained; for this purpose, a lower percentage dispersion threshold, for example $2 \%$, is required, above which the 


\section{International Advanced Research Journal in Science, Engineering and Technology}

Vol. 8, Issue 4, April 2021

\section{DOI: $10.17148 / I A R J S E T .2021 .8417$}

principal components are considered. The other principal components, with a contribution of less than $2 \%$, are neglected because they correspond to small variations in the initial variables.

For example, fig. 6 shows the establishment of the principal components (PC) for the experimental series containing 9 functional variables mentioned in the graphs from the 40 tests in the urban environment, respectively in the extraurban environment. For a good readability, on the axis of the ordinates from fig. 6 the percentage values are given and not the absolute values of the contribution of each principal component to the total dispersion. Fig. 6a shows that, for a threshold of $2 \%$, in the urban environment the engine torque $M_{e}$ requires the consideration of 14 principal components, the other variables requiring fewer PC; consequently, the highest value is adopted in the urban environment, ie $14 \mathrm{PC}$, the other 26 being neglected (the difference up to 40 in the number of tests). In this case the reduction of the number of data is almost 2.85 times, from 1440000 values to 504000 values, as shown in fig. 10a.

Fig. $6 \mathrm{~b}$ shows that, for a threshold of $2 \%$, in the extraurban environment the motor moment $M_{e}$ requires the consideration of 5 principal components, the other variables requiring less PC; consequently, in the extraurban environment the highest value is adopted, ie $5 \mathrm{PC}$, the other 35 being neglected. In this case the reduction of the number of data is 8 times, from 1440000 values to 180000 values, as shown in fig. $6 \mathrm{~b}$.

Therefore, when traveling outside the city (in the extraurban environment), it is possible to reduce the number of data more than in the urban environment. This means that the evolution trends of the targeted process (here the dynamics of the vehicle) are better highlighted, with a higher concentration of data in fewer directions/axes.

Determining the number of principal components, 9 variables, Ford Focus car
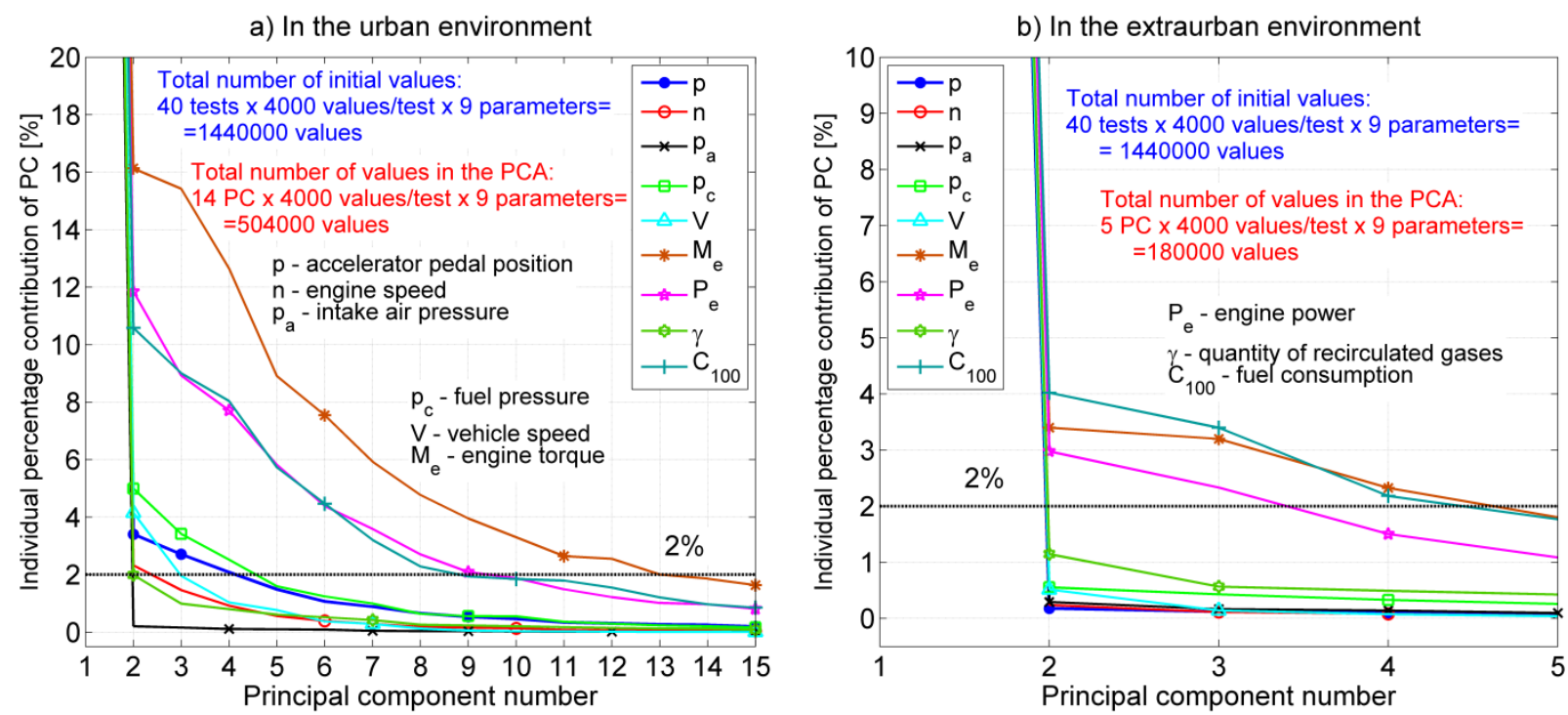

Fig. 6 Determining the number of principal components, Ford Focus car

Fig. 7 verifies the correctness of adopting the number of principal components for the engine moment from an experimental test in the extraurban environment. As it turns out, the adoption of a number of 5 main components for the restoration of the experimental series ensures an error of less than $2 \%$ as a value imposed for reasons of accuracy. 
DOI: $10.17148 /$ IARJSET.2021.8417

\section{Checking the number of principal components, FE25 test in the extraurban environment, Ford Focus car}

a) With 2 principal components

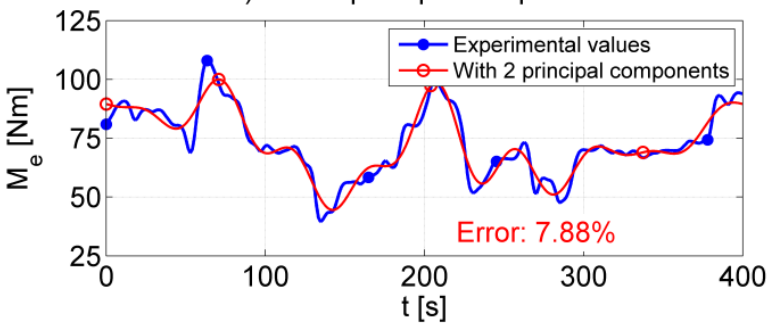

c) With 4 principal components

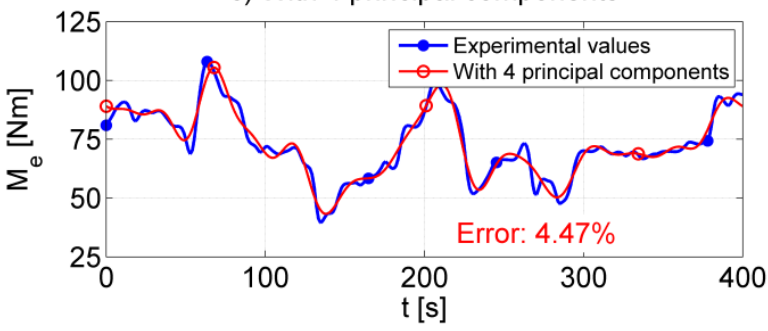

b) With 3 principal components

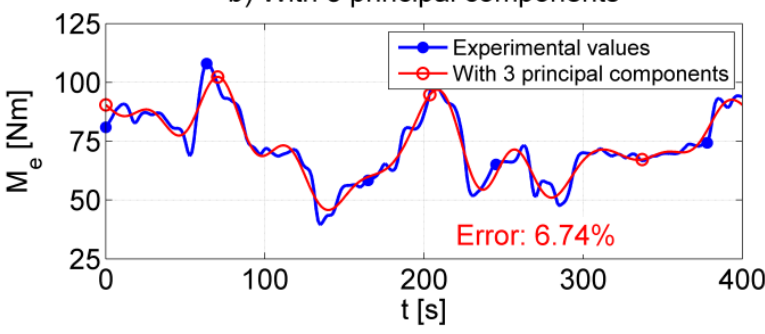

d) With 5 principal components

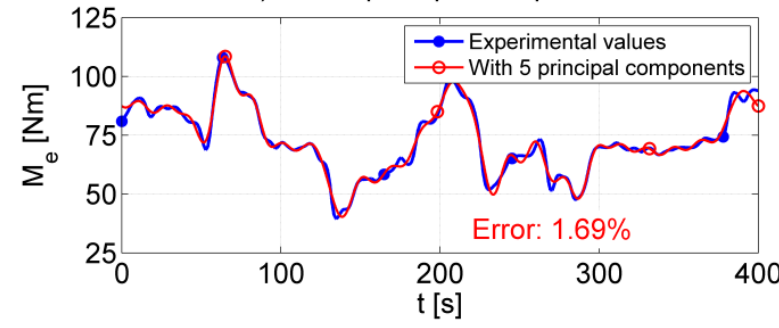

Fig. 7 Checking the correctness of adopting the number of principal components, Ford Focus car.

\section{Cluster analysis}

Cluster Analysis (CA), or group analysis, is part of the category of multivariable techniques that ensure the grouping of data according to what unites them (what they have in common), so based on their similarity, as opposed to analysis discriminatory (subsequently treated), which concerns what distinguishes the data considered [2]. Cluster analysis allows data to be arranged in groups/clusters with the same characteristics, thus facilitating various subsequent analyzes. In the case of experimental data, the same characteristics for a cluster mean the same distance from the average value; as previously stated, distance is a basic notion in multivariable statistics, also called geostatistics.

There are several methods for establishing clusters, the most used being the following two:

- partitioning methods, which ensure the distribution by groups using an optimal criterion, most often aiming at minimizing the dispersion $\sigma^{2}$ (with $\sigma$ standard deviation):

$$
\sigma_{o p t}^{2}=\min _{P} \sum_{p \in P} \sum_{i \in p}\|i-c\|^{2}
$$

in which case the Euclidean distance is $d^{2}(i, p)=\|i-p\|^{2}$, and the partition $P$ has $p$ clusters with centers $c$.

- hierarchical methods, which are based on minimizing the dissimilarity matrix, which is built using the distances between the values in each cluster $i$ and its center but also the distances between the centers $c_{i}$ and $c_{j}$ of two clusters, the second having $j$ values:

$$
d_{\text {opt }}=\frac{|i| \cdot|j|}{|i|+|j|}\left\|c_{i}-c_{j}\right\|^{2}
$$

in which case the graph called the dendrogram is obtained. It should be noted that the center of the cluster is targeted in the case of the " $k$ - means" algorithm, but the relation can also be applied for the singular points as in the case of the " $k$ - nn" algorithm.

Fig. 8 and fig. 9 show the analysis on clusters by partitioned methods in the case of the dependence of the speed of vehicle $V$ on the position of the accelerator pedal $p$ when moving the Ford Focus car in the extraurban and in the urban environment, respectively. The two graphs show, for each of the 5 clusters, the respective center and its coordinates. The graphs in fig. 8 and fig. 9 show a good separation on clusters in both cases. Also, these graphs confirm the lower speeds in the urban environment, in addition they confirm the lower presses on the accelerator pedal. 


\section{International Advanced Research Journal in Science, Engineering and Technology}

Vol. 8, Issue 4, April 2021

DOI: $10.17148 /$ IARJSET.2021.8417

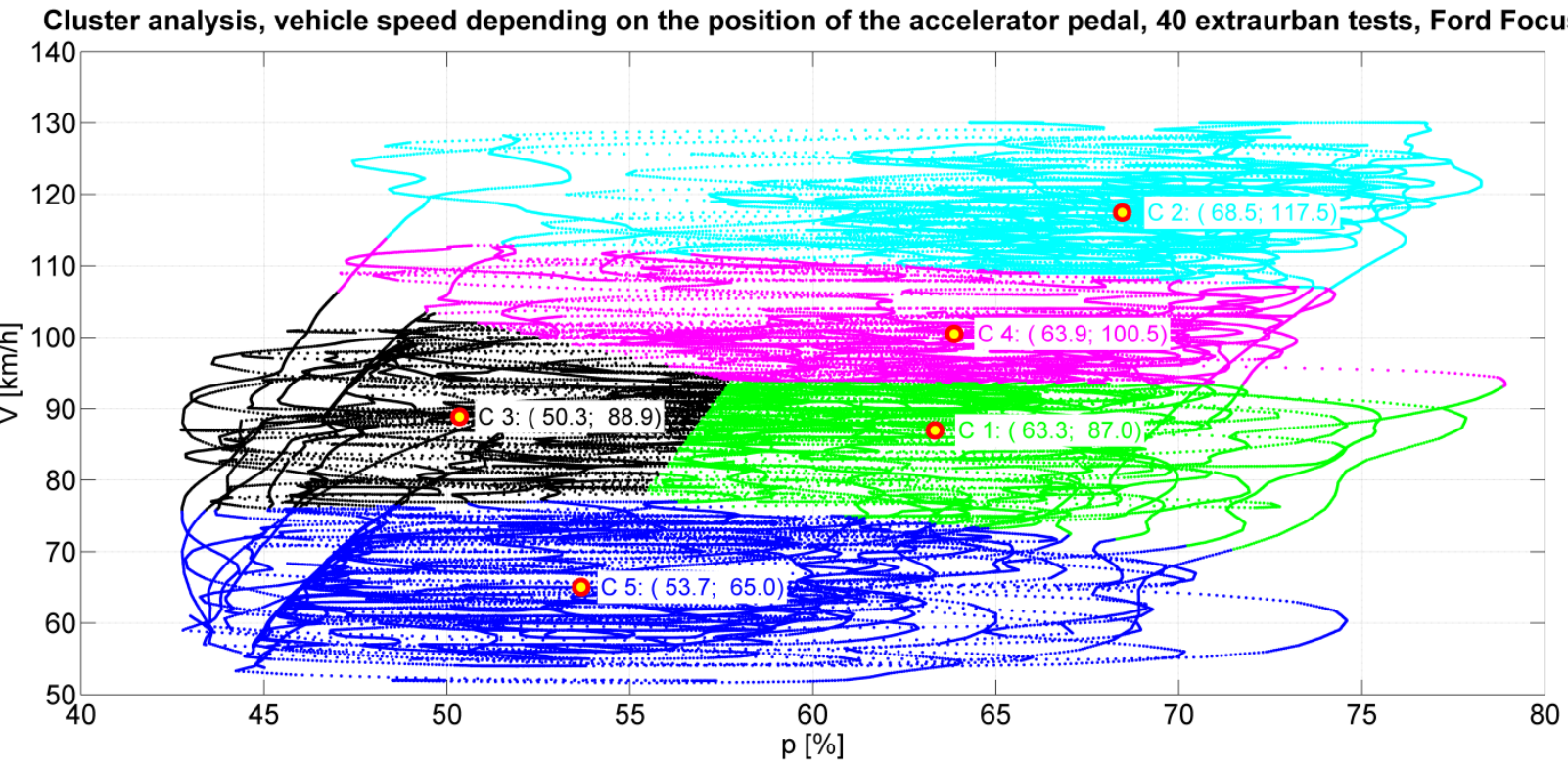

Fig. 8 Cluster analysis, vehicle speed depending of the accelerator pedal, in extraurban environment

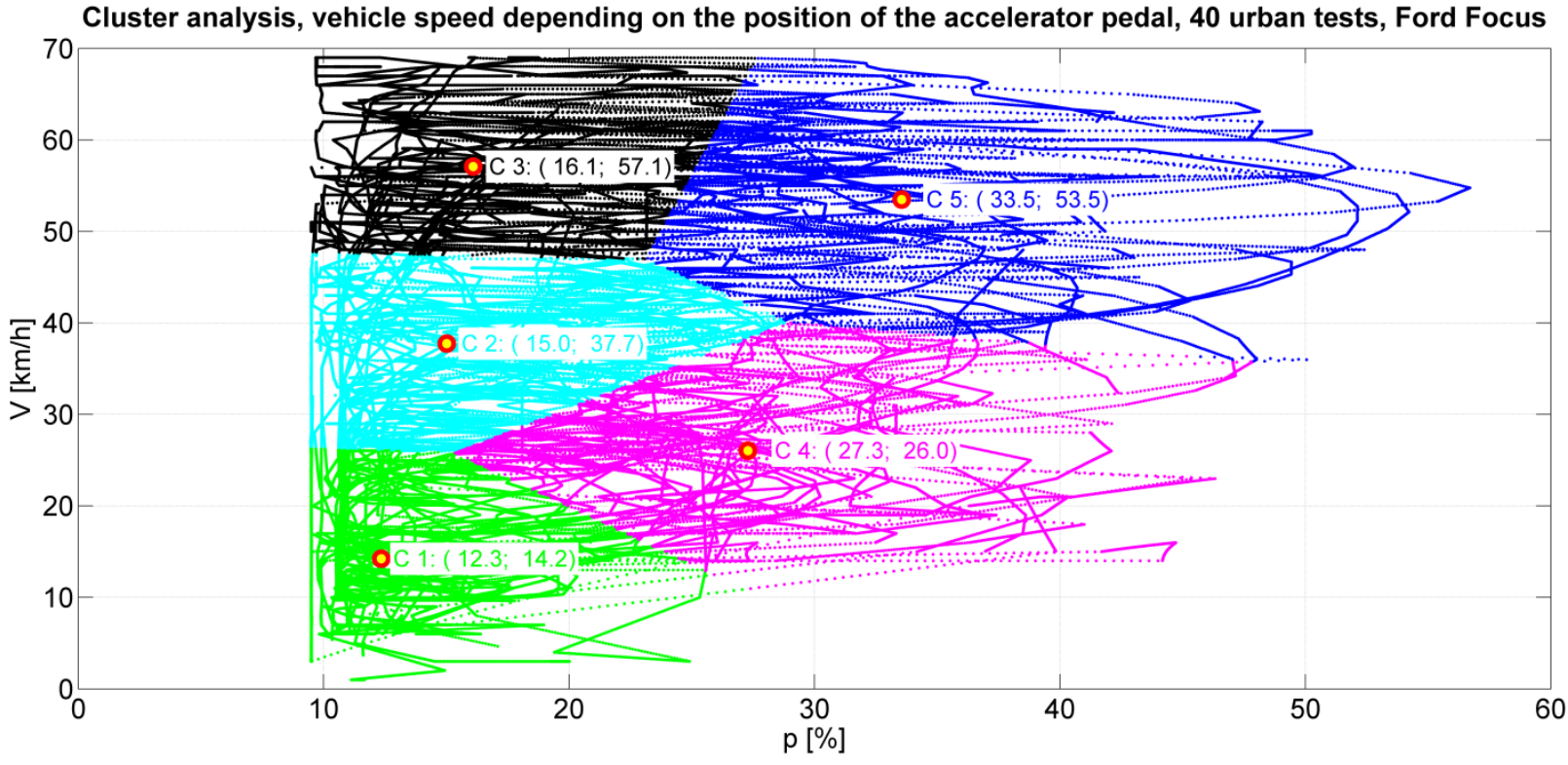

Fig. 9 Cluster analysis, vehicle speed depending of the accelerator pedal, in urban environment

Fig. 10 shows an example with the analysis on clusters by hierarchical methods, which aims to establish the dendrogram and silhouette. The target size is the speed of the vehicle, based on which the 6 clusters in the upper graphs where the dendrograms are shown are established. The graphs below show the values of the silhouettes when driving the VW Touareg II 3.0 V6 TDI in urban and extraurban areas.

The dendrograms in the upper graphs show the similarity of Euclidean distances for different clusters; for example, in the urban environment (fig. 10a) clusters 1 and 2 are characterized by the smallest distance, so they are the closest from a statistical point of view. Another example, from fig. $10 \mathrm{~b}$ it is found that clusters 5 and 6 are the closest statistically. The consequences of these aspects are found when processing experimental data; for example, small errors in establishing mathematical models are obtained in statistically close groups.

The lower graphs in fig. 10 show the silhouette of the speed in the case of the 6 established groups. A silhouette of a cluster has a length (horizontally) and a width (vertically); the first means how the data is grouped (an approximation of the unit value shows a good grouping/separation by groups), and the second shows the amount of data (a large width indicates a large number of data). For example, in cluster 1 of fig. 10c the data are very scattered (the 


\section{DOI: $10.17148 / I A R J S E T .2021 .8417$}

values of the silhouette are far from the unitary one), and the number of data is large (the width of the silhouette is large).

The graphs below show that when travelling in extraurban areas, the grouping of data is better, the values of the silhouette being higher than those in urban areas. This means that in extraurban mode the mathematical models established for the dynamics of the vehicle have a higher accuracy and a higher degree of prediction.

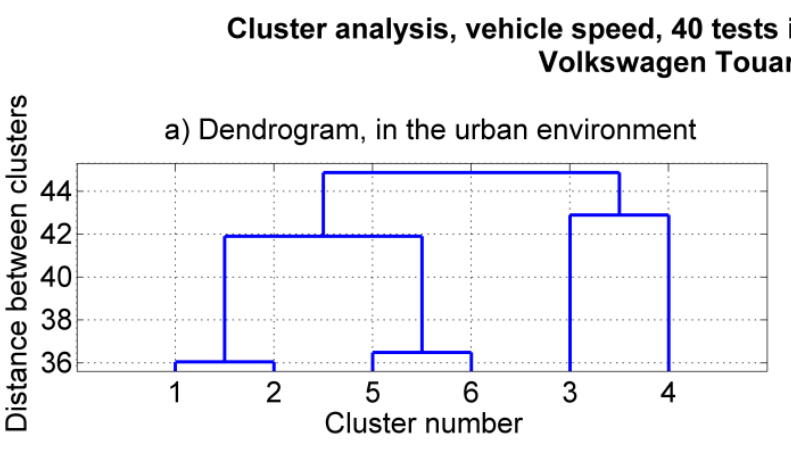

c) Silhouette, in the urban environment

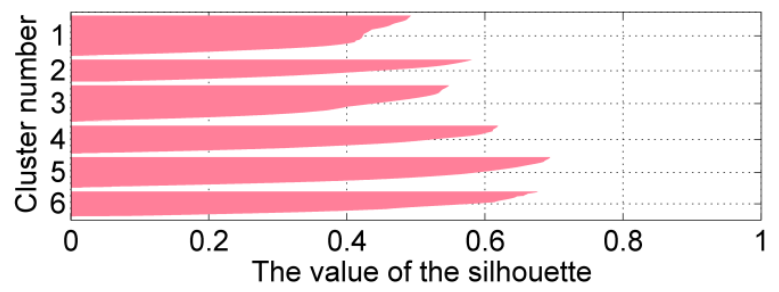

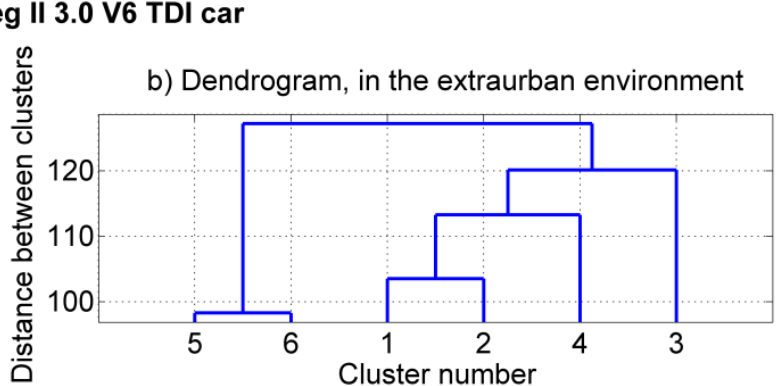

d) Silhouette, in the extraurban environment

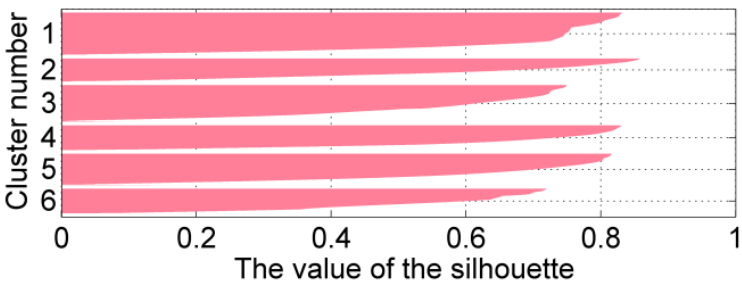

Fig. 10 Cluster analysis by ranking methods, vehicle speed, VW Touareg II car

\section{Discriminatory analysis}

A discriminatory approach to the previous one (cluster/group analysis) has a discriminatory analysis (DA), introduced by the mathematician Fisher, which establishes what is not common to the analyzed data, so what distinguishes them from each other. Discriminatory analysis is also based on grouping data, but the groups obtained are usually called classes, and the principal components become vectors. In addition, both types of analysis (cluster analysis and AD) use classification algorithms for class/group classification, so it uses concepts of decision theory.

Classification is based on specific criteria for discriminatory analysis and possibly imposing certain additional conditions. Some of these criteria are presented in fig. 15 (Kozinec, Anderson, Bayes, Anderson-Bahadur criteria), which shows a separation by classes according to some rules of linear or quadratic decision, based on the objectively imposed function. As an example, the following is Fisher's criterion, which maximizes the ratio between the mean values of the value series and the dispersion of the entire data matrix that forms the target sample. As it can be seen from fig. 15a, the criterion belongs to the linear discriminatory analysis (LDA - Linear Discriminant Analysis, respectively FLDA - Fisher Linear Discriminant Analysis); instead, the other cases in fig. 15 belong to the Quadratic Discriminant Analysis (QDA), which uses several criteria [1, 5].

According to fig. 15a, the discriminant function of Fisher's criterion has a linear shape of the type:

$$
D=w_{1} x_{1}+w_{2} x_{2}+\ldots+w_{n} x_{n}
$$

where $x_{(\cdot)}$ represents the vectors, and $w_{(\cdot)}$ the coefficients of the discriminant function; often we operate with standardized vectors $z_{(\cdot)}$, ie with variables that have zero mean and unit standard deviation (that's why negative values also appear).

Expression (7) can be written in matrix form such as:

$$
D=\mathbf{w}^{T} \mathbf{x}
$$

where " $T$ " represents the symbol of mathematical transposition operation. 


\section{International Advanced Research Journal in Science, Engineering and Technology}

Vol. 8, Issue 4, April 2021

\section{DOI: $10.17148 /$ IARJSET.2021.8417}

Criteria from the decision theory used to establish classes in discriminatory analysis

a) Fisher criterion, Kozinec algorithm

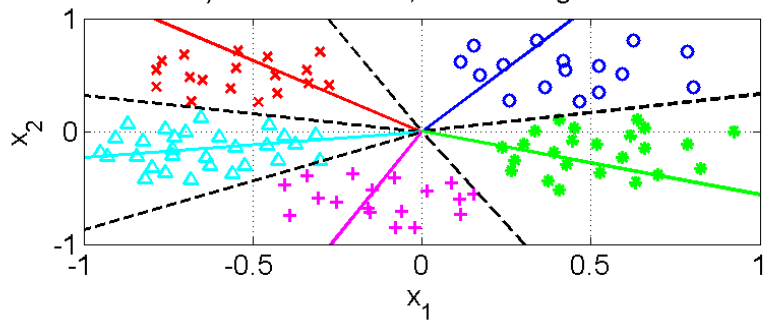

c) Bayes criterion

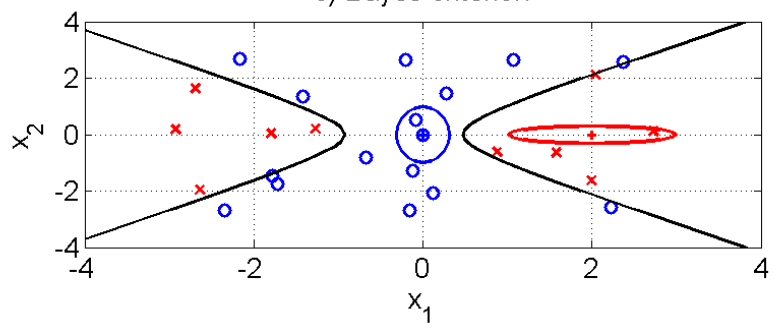

b) Anderson`s optimal criterion

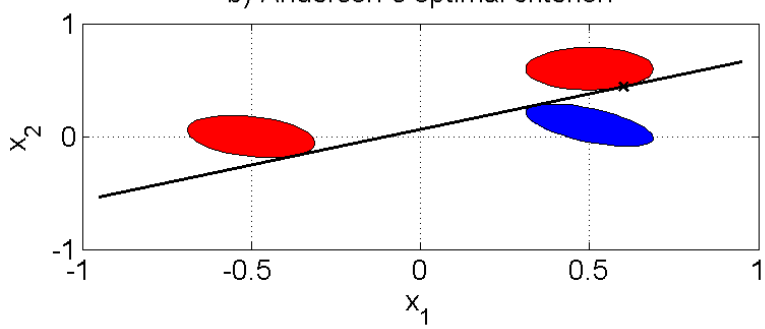

d) Anderson-Bahadur criterion

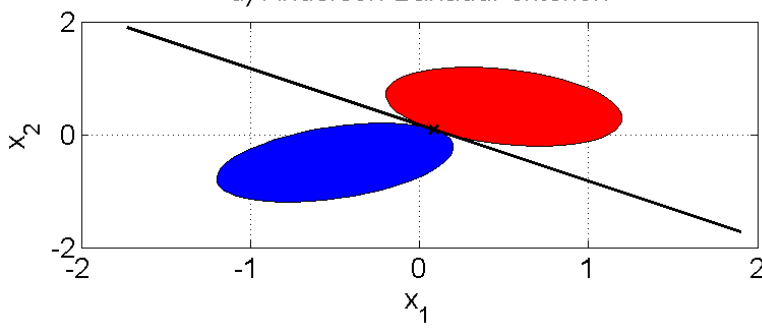

Fig. 11 Criteria from decision theory used to establish classes in discriminatory analysis

Fig. 12 shows the discriminatory analysis for vehicle speed from 3 experimental tests in the extraurban environment of the VW Touareg II car (VE notation), which aimed to establish 6 classes.

As shown in the graph, it was possible to separate the classes with the values that differentiate the three tests, even if there are areas with interfering values as seen in fig. 13.

\section{Discriminatory analysis, 6 classes, vehicle speed, 3 tests in the extraurban environment, Volkswagen Touareg II 3.0 V6 TDI car}

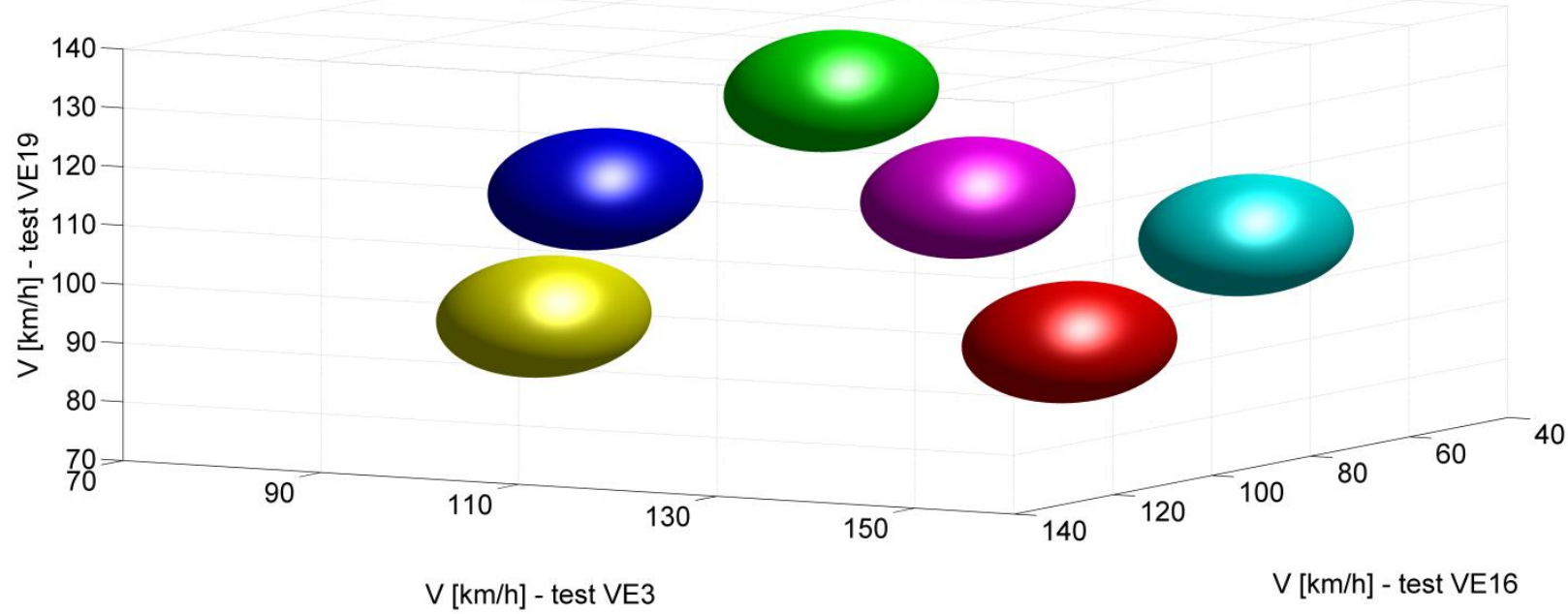

Fig. 12 Discriminatory analysis, 6 classes, vehicle speed, 3 tests, extraurban environment, VW Touareg II car 
DOI: $10.17148 /$ IARJSET.2021.8417

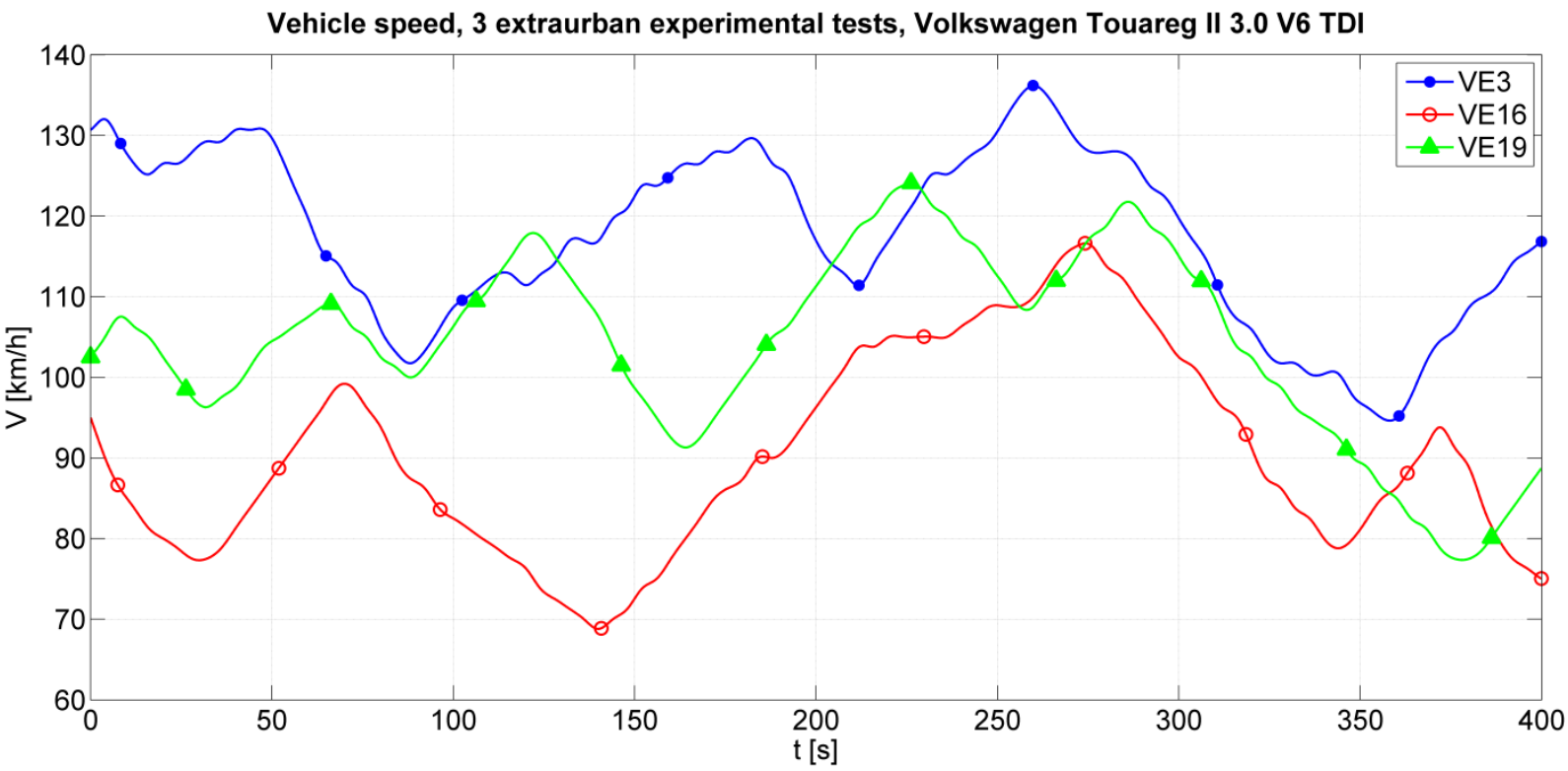

Fig. 13 Vehicle speed, 3 experimental tests in the extraurban environment of the VW Touareg II car

It should be noted that classes with different values cannot always be completely separated, as noted in fig. 14 for three speed tests in the urban environment (VU notation), classes C4 and C5 having common areas.

For this case, in fig. 15 are presented the values of the speed of the 3 experimental samples from the urban environment (VU1, VU15, VU33).

\section{Discriminatory analysis, 6 classes, vehicle speed, 3 tests in the urban environment, Volkswagen Touareg II 3.0 V6 TDI car}

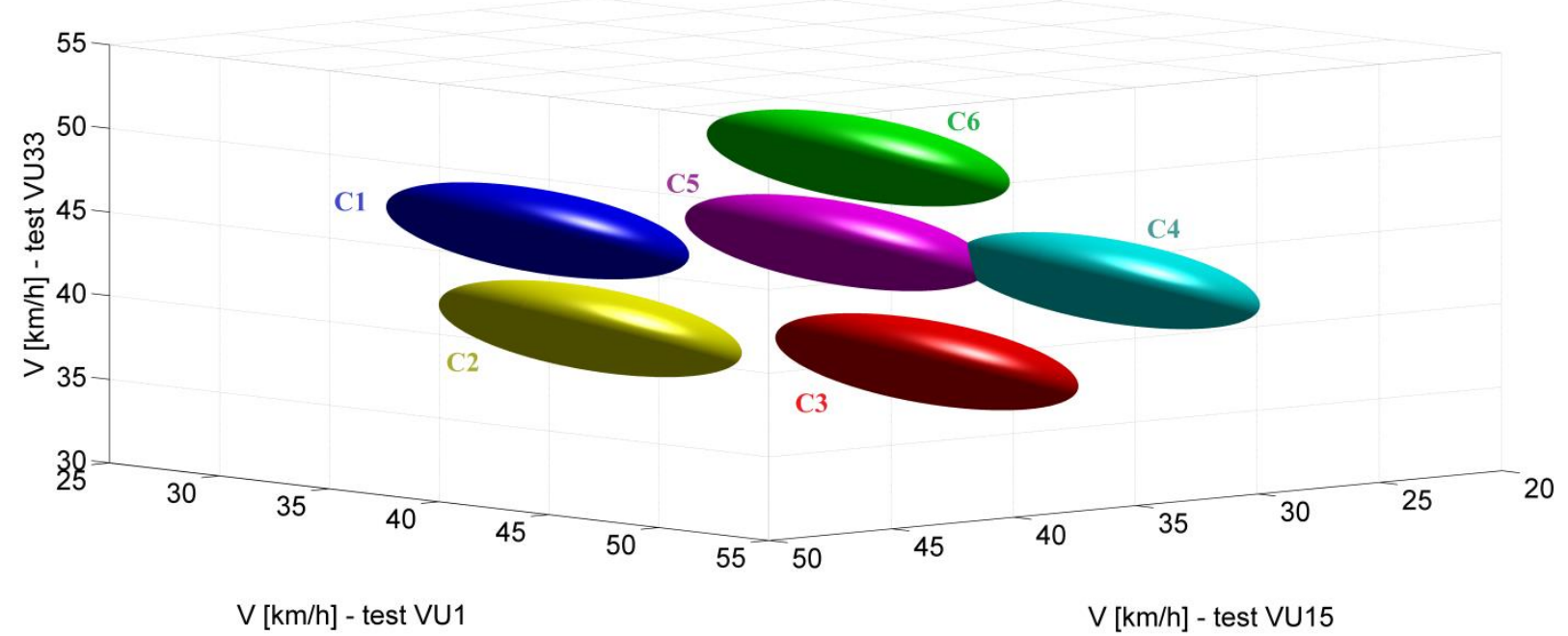

Fig. 14 Discriminatory analysis, 6 classes, vehicle speed, 3 tests, urban environment, VW Touareg II car

It should also be mentioned that if the classes obtained have normal distributions, so if Gaussian mixtures are deduced, then each mixture $k$ has the probability density:

$$
f_{k}(x)=\frac{1}{(2 \pi)^{p / 2}\left|C_{k}\right|^{1 / 2}} \exp \left[-\frac{1}{2}\left(x-\mu_{k}\right)^{T} C_{k}^{-1}\left(x-\mu_{k}\right)\right]
$$

with $C_{k}$ covariance matrix and $m_{k}$ media of the respective class. In such cases, there is a Gaussian Linear Discriminant Analysis (GLDA). 
DOI: $10.17148 / I A R J S E T .2021 .8417$

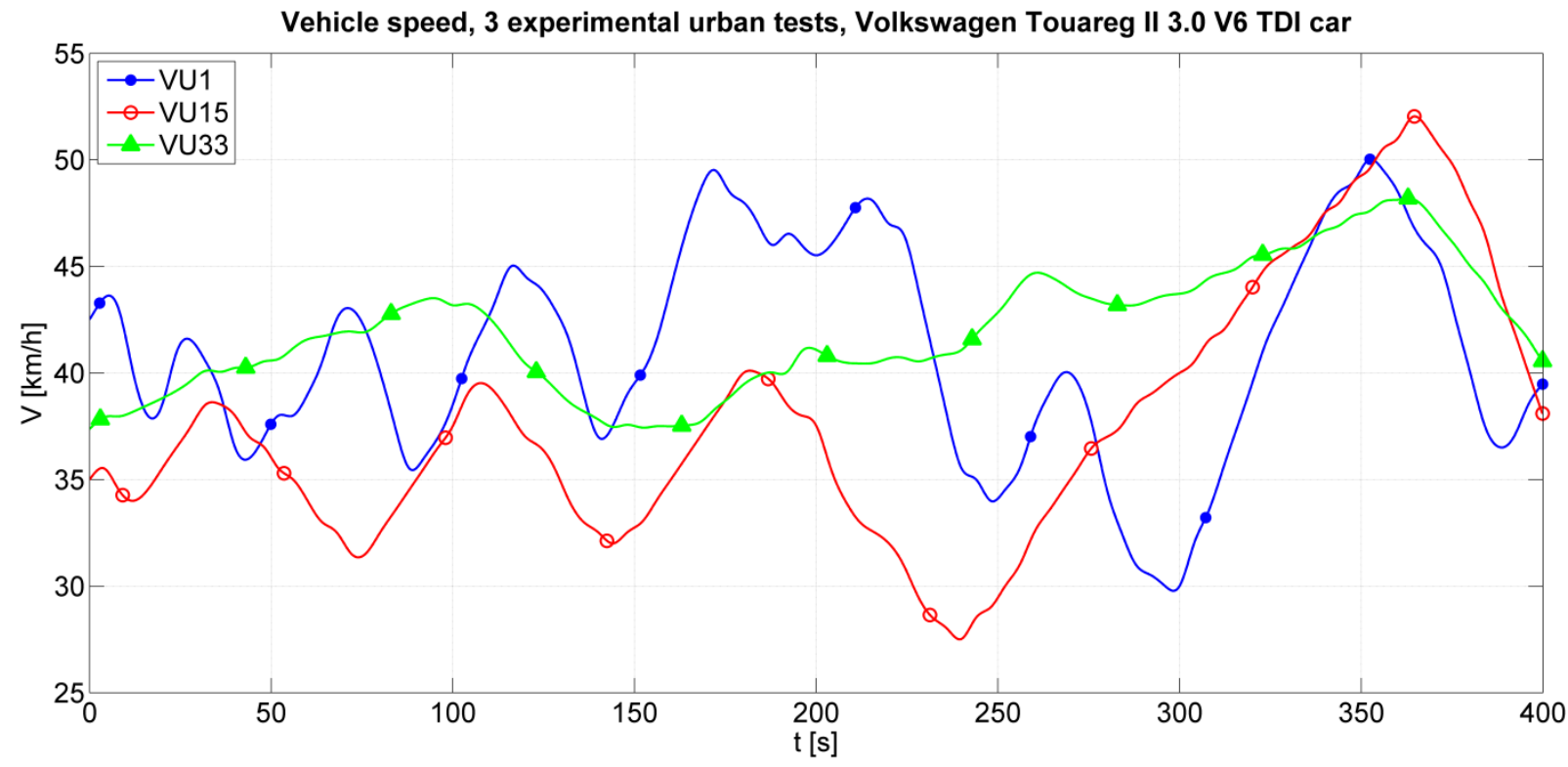

Fig. 15 Vehicle speed, 3 experimental tests in the urban environment of the VW Touareg II car

\section{IV.CONCLUSION}

The study, part of which is presented here, showed that when travelling in extraurban areas, vehicle speeds, loads and engine speeds are higher, and accelerations are lower than when travelling in urban areas. Also, in the extraurban environment the fuel consumption is lower than in the urban environment. At the same time, energy efficiency, which takes into account both the dynamism and the fuel saving of the vehicle, is superior in the extraurban environment.

The study of vehicle dynamics with multivariable statistical techniques showed that when travelling in extraurban areas, the spatial correlation is more pronounced than in urban areas. Also, in the extraurban environment, the number of available data can be reduced more, and the separation by clusters and classes is better, which means that the evolution trends are clearer than in the urban environment.

\section{REFERENCES}

[1]. S. Balakrishnama, "Linear Discriminant Analysis", Institute for Signal and Information Processing, Mississipi State University, 1995.

[2]. V. Hietanen, "Use of Cluster Analysis in Automation Technology", Helsinki University of Technology, 2002.

[3]. H. Hyötyniemi, "Multivariate Statistical Methods in Systems Engineering". Report 112, Helsinki University of Technology, 1998.

[4]. A. Ilin, "Principal Component Analysis", AS-116.140 Schedule, Helsinki University of Technology, 2002.

[5]. Md Rakibul Islam and Sifat Hasan, "Integration of Mechanical Systems with IoT", International Advanced Research Journal in Science, Engineering and Technology (IARJSET), Vol. 8, Issue 1, January 2021, pg. 43-50.

[6]. S. Savada, "Global Spatial Autocorrelation Indices-Moran's I, Geary's C and the General Cross-Product Statistic", Departament of Geography, University of Otawa, 2003, http://www.lpc.uottawa.ca/ publications/moransi/moran.htm.

[7]. D. Unwin, "GIS, spatial analysis and spatial statistics", University of London, 1996.

[8]. J. Zuur, "Spatial autocorrelation", Academic Press, San Diego, 2000.

[9]. L. Barothi, D. Voicu, R.-M. Stoica and M. Singureanu, "Recording of parameters characteristic to engine and vehicle in order to validate a simulation model for fuel consumption", 5th International Scientific Conference SEA-CONF 2019, 17-18 May 2019, Mircea cel Batran Naval Academy, Constanta, Romania, IOP Conf. Series: Journal of Physics: Conf. Series 1297 (2019) 012030 IOP Publishing, doi:10.1088/1742$6596 / 1297 / 1 / 012030$

[10]. I. Lespezeanu, R.M. Stoica, R. Vilau, D. Sava and I. Copae, “Comparative Study Regarding the Operation of a Car's Internal Combustion Engine in Urban and ExtraUrban Environment", The 30th SIAR International Congress of Automotive and Transport Engineering, pg. 177-187, oct. 2019, published 2020, DOI: 10.1007/978-3-030-32564-0_21

[11]. R. Vilău, I. Lespezeanu and M. Singureanu, "Considerations regarding the influence of inappropriate electronic throttle unit operation on pollutant emissions of internal combustion engines", 5th International Scientific Conference SEA-CONF 2019, IOP Conf. Series: Journal of Physics: Conf. Series 1297 (2019) 012032, IOP Publishing, doi:10.1088/1742-6596/1297/1/012032 